Reihe Informatik. TR-2007-011

\title{
Studying Malicious Websites and the Underground Economy on the Chinese Web
}

\author{
Jianwei Zhuge ${ }^{1}$, Thorsten Holz ${ }^{2}$, Chengyu Song ${ }^{1}$, \\ Jinpeng Guo ${ }^{1}$, Xinhui Han ${ }^{1}$, and Wei Zou ${ }^{1}$ \\ ${ }^{1}$ Peking University \\ Institute of Computer Science \\ ${ }^{2}$ University of Mannheim \\ Laboratory for \\ and Technology \\ Beijing, China \\ Dependable Distributed Systems \\ Mannheim, Germany
}

December 3, 2007

\begin{abstract}
The World Wide Web gains more and more popularity within China with more than 1.31 million websites on the Chinese Web in June 2007. Driven by the economic profits, cyber criminals are on the rise and use the Web to exploit innocent users. In fact, a real underground black market with thousand of participants has developed which brings together malicious users who trade exploits, malware, virtual assets, stolen credentials, and more. In this paper, we provide a detailed overview of this underground black market and present a model to describe the market. We substantiate our model with the help of measurement results within the Chinese Web. First, we show that the amount of virtual assets traded on this underground market is huge. Second, our research proofs that a significant amount of websites within China's part of the Web are malicious: our measurements reveal that about $1.49 \%$ of the examined sites contain some kind of malicious content.
\end{abstract}

\section{Introduction}

The World Wide Web (WWW) becomes more and more important each day within China. A large number of Chinese Internet users have enjoyed the convenience and flexibility the Web brought them, from searching for information, online entertainment to e-business, and e-finance [2]. According to the latest Alexa Global top 500 websites list [1] (32 Chinese websites are in the list), there are four types of most successful and best known sites within the Chinese Web: the first type of websites are search engines, including Baidu, Google.cn, Yahoo! China, Tencent's SoSo and Suhu's Sogou. Among them, Baidu and Google are the most popular ones. The second category contains portals and navigation sites. Among the seven sites belonging to this category, Tencent's QQ, Sina, NetEase 163, Sohu and TOM are listed in the top ten Chinese websites. The third type of sites is related to e-business: the Taobao C2C (customer-to-customer) online business platform and the Alibaba B2B (business-to-business) platform both operated by Alibaba group - are the most well-known within the Chinese Web. The last type of sites contains sites in the area of online entertainment and virtual personal space, including YouTube-like sites such as 56.com, toodou, ku6, several myspace-like sites such as poco, bokee, and others.

But there is also the other side of the coin: targeting the virtual assets owned by the normal Chinese Internet users, malicious attackers, so called blackhats, discover the Web as a new venue for making money by exploiting innocent users. A common theme is to inject malicious code into a bought or cracked website. The injected code exploits an unpatched client-side vulnerability within the visiting web-browser or related application. Each time a user with a vulnerable version of a browser or related application visits 
this site, his machine is compromised and some kind of malware is automatically installed. This kind of attack is also called drive-by-download attack. The malware is quite often some kind of Trojan Horse that searches for valuable information on the victim's machine and then sends the information back to the attacker, who in turn can sell this virtual good to other attackers or innocent users.

In this paper, we study this phenomenon on the Chinese Web in more detail. We introduce a model to describe the underground economy which drives the malicious websites phenomenon and the individual actors within this ecosystem. The model describes the underground economy that we have studied within the Chinese Web and thus some aspects of it are specific to China. However, our model can also be used to describe the market for malicious tools and stolen goods for other parts of the Web or the Web as a whole. Our measurements show that there are thousand of participants within this market and there are strong relations between the underground black market and the public virtual assets trading. Furthermore, we also measure the extent of malicious websites within China's part of the Web with the help of client honeypots. During our measurement of about 145,000 of the most commonly visited websites on the Chinese web, we found 2,149 , i.e., $1.49 \%$ of them, containing malicious content. We also performed redirection link analysis which can disclose the relationship between the malicious websites and the hosts of web-based exploits, as well as the top active exploiters. Finally, we examined the detection rates of the major anti-virus engines against the specific threat faced by the normal Chinese Internet users, which shows the severe situation modern anti-virus software has to detect latest threats.

This paper is outlined as follows: In Section 2, we provide an overview of related work in the area of malicious websites and the underground black market. We introduce a model to describe the underground economy and the different actors within this ecosystem, together with a case study, in Section 3 . In Section 4, we show different mechanisms used by attackers to create malicious websites which we found by studying a large amount of actual attacks on the Chinese Web. The results of a measurement study on the underground black market and malicious websites within the Chinese Web are presented in Section 5. Finally, we conclude the paper in Section 6 with an overview of future research.

\section{Related Work}

The work closest related to ours is a study on the underground black market by Franklin et al. [5]. The authors study a large number of underground IRC channels and keep track of advertisements for virtual goods. Based on the collected information, they examine the size of the underground black market, the number of virtual goods traded, and similar characteristics. Our work is orthogonal to the work by Franklin et al.: We study the aspects of the underground market visible as part of the World Wide Web. We examine the relationship between the individual actors within the market and also study the size of the actual market via different metrics. Furthermore, we also introduce a model for the underground economy and substantiate our model with the help of real-world data collected on the Chinese Web. Other studies orthogonal to ours focus on different aspects of the underground economy [3,11].

The first research efforts to analyze malicious websites were published by Wang et al. (HoneyMonkey [12]) and Moshchuk et al. [6]. The key idea in both projects is to automatically browse the Web and analyze all content in order to detect sites that try to infect an unprotected user. Both projects show that such an effort is viable and they could detect malicious sites in an automated way. We extend the original idea by combing ideas from both projects in order to achieve a more scalable solution that still has the capability of detecting unknown exploits. The most detailed overview of the threat posed by malicious websites is given by Provos et al. [8]. Using Google's cache of crawled websites, they analyzed the extend of this threat and could give for the first time numbers showing the maliciousness of a significant part of the Web. We focus on the Chinese Web and show that this part of the Web also hosts a significant amount of malicious content. In addition, we also analyze parts of the Web that are not easily reachable by Google, e.g., the virtual goods offered at the Taobao online business platform or the advertisements posted at the Baidu Post Bar. The Honeynet Project has released several papers which deal with the phenomenon of malicious websites. One of the papers deals with attacks against web applications [10], while the other focuses on client-side honeypots [9]. We extend the original idea of client honeypots by combining two techniques that allow us to significantly speed-up the analysis platform. 


\section{Underground Economy Model}

\subsection{Modeling the Individual Actors}

In this section, we introduce a model to describe the interaction between different actors within the underground black market. We explain the economic aspects of the phenomenon and for each actor, we illustrate their role, what kind of information / goods they trade, and what the common price for such goods / services is. This model is adapted to the Chinese Internet, since the social aspects within China enable a unique ecosystem. However, our model can also be extended to describe the blackhat underground economy in other countries and is not specific to China per se.

\subsubsection{Virus Writers}

Virus Writers are malicious Internet users driven by economic profits. They have a certain degree of technical background of computer networks and programming skills, e.g., they are able to find vulnerabilities in software (so called 0 -day vulnerabilities) themselves, or they use recently public disclosed vulnerabilities and the corresponding exploits. Furthermore, these actors have the technical skills to develop their own exploits, or Trojans based on the original vulnerability reports and available exploit codes. Then they sell their tools and malware for making money, and provide evasion service to their customers.

To find potential customers, they post advertisements on the underground black market. The markets are typically online discussion boards, so called bulletin board systems, within the World Wide Web that are used for discussions. Furthermore, the boards provide a platform for sellers and buyers of this kind of resources. In addition, experienced Virus Writers often release some limited version of their tools or malware for free, in order to raise their status and reputation in the blackhat community. Moreover, obliques and imputations between the competitors are very common within these online communities.

We searched within the underground black market and found the following prices for typical "services" within this market: the market price of a Trojan is between tens to thousands Renminbi (RMB), and a package of 0-day powerful Trojan generator and evasion service can be up to several ten thousands RMB. 10 RMB is as of November 2007 equivalent to \$1.34 US dollar. This means that such software has a certain value and Virus Writers have the incentive to invest time and knowledge into this area.

\subsubsection{Website Masters/Crackers}

The second actor within the underground market are Website Masters and Website Crackers. The administrators of certain personal websites attract visitors with the help of free goodies, e.g., free movies, music, software, or tools. These websites often betray their visitors: they sell the traffic (i.e., website visits) of their websites to Envelopes Stealers (see next section) by hosting the Web-based Trojans. This means that innocent websites visitors are redirected via these malicious websites to other sites that then attack the victims. If the attack is successful, a piece of malware is installed on the victim's machine.

Website Crackers hack into well-known, but unsafe websites by exploiting vulnerabilities that exist on these sites. Via the command line access on the compromised machines, they then redirect the traffic for this website to another malicious machine, i.e., they then sell the traffic of their victim's website. Our research revealed a market price of about $40-60 \mathrm{RMB}$ per ten thousand IP visits.

\subsubsection{Envelopes Stealers}

"Envelopes" is a jargon word used in the underground market which means the stolen pair of account and password, i.e., the credentials for a given site. We will use this term in the following throughout the paper. Envelopes Stealers have very limited technical knowledge and know only little about the technology and hacking skills. Typically, they buy ready-to-use Trojans or even malware generators from Virus Writers, and website traffic from Website Masters/Crackers. All they need to do is to create a Web-based Trojan network from which they can harvest envelopes: they combine a Web-based Trojan (which exploits vulnerabilities in the browsers, underlying components, or related applications) with a conventional Trojan for stealing certain envelopes and link the generated Trojan to the bought websites. 
They then sell the harvested envelopes to Virtual Asset Stealers, which we introduce in the next section. We found that the market price of an envelope varies from some Jiao to tens of RMB. They also sell access to the compromised machines, which are called flesh chicken (because "chicken" has the same pronunciation as "machine" in Chinese), in the underground market. The market price of a "flesh chicken" is between $0.1-10 \mathrm{RMB}$, thus it is rather cheap for an attacker to control a compromised machine.

\subsubsection{Virtual Asset Stealers}

Virtual Asset Stealers do not have any technical knowledge about hacking and programming, but they have a rather good understanding of the underground market itself. Typically, they know which online games are currently popular and which virtual asset (for example equipment in games) can be sold for a good price. They buy envelopes from the Envelopes Stealers, log-in to the online games or QQ accounts to steal valuable virtual assets like game equipments or QQ coins. Besides these monetary goods, these actors also steal other valuable, virtual goods including "beautiful" QQ accounts, i.e., accounts with a short name, or a name that can be easily remembered.

After getting access to the virtual assets, they commonly sell them to others. Besides a prospering market for QQ accounts, we also found evidence that they sell other virtual assets like powerful equipments for various online games. The market price varies for each virtual good, e.g., game equipments is typically sold for $10-10 \mathrm{~K} \mathrm{RMB/equipment,} \mathrm{whereas} 1$ QQ coin commonly costs $0.2-0.3 \mathrm{RMB}$. It is interesting to observe that the official exchange rate by the vendor Tencent is 1 RMB for 1 QQ coin. During the "Super Voice Girls" competition, an annual national singing contest, the black market price rose to $0.3-0.5 \mathrm{RMB}$ since enthusiastic fans were seeking for QQ coins to vote for their favorite contestants.

\subsubsection{Virtual Asset Sellers}

Another party within the whole underground are the Virtual Asset Sellers, which can also be (but not need to be) Virtual Asset Stealers. They contribute to the circulation section of the industry chain by setting up virtual shops on the World Wide Web. These shops can be commonly found at Taobao, Tencent's PaiPai, and eBay, the three biggest online business platforms within China.

Our research shows that the Virtual Asset Sellers usually buy the virtual assets from the underground market on bulletin board systems with a very low price. They then sell them to Players on the public marketplaces, making profit due to the price difference between buying and selling. For example, they typically buy QQ coins on bulletin boards and then sell the coins for $0.5-0.8$ RMB on Taobao, making a certain profit with each transaction.

\subsubsection{Players}

The sixth actor within our economic model are the Players, who are enthusiastic online games players (or QQ users), often spending large amounts of money on the virtual assets. The Players are commonly male teenagers who dispense their parents' money for fun on the World Wide Web and in online games. They are the foundation of the whole underground market since they stimulate demand for all virtual goods and drive the market.

\subsection{Market Interaction}

In Figure 1, we provide an overview of the interaction of the individual actors within the underground market. The business between the six different actors within the ecosystem takes place in different locations. For example, businesses between Envelopes Stealers, Virus Writers, and Website Masters/Crackers takes place in the underground black market on different kinds of bulletin board systems. These systems also provide a market place for Envelopes Stealers and Virtual Asset Stealers as well. On the other hand, the circulation of virtual assets is open on the World Wide Web. This is due to the fact that they need normal players to find them easily and there are very weak controls on the circulation of stolen virtual assets in China. 


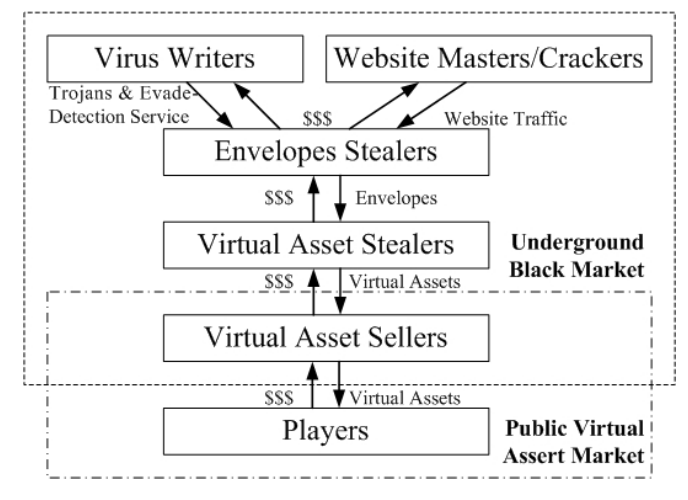

Figure 1: Interaction of the individual actors within the underground market on the Chinese Web

As noted previously, the underground market commonly uses bulletin board systems to connect the individual actors. One of the most prominent places for such markets within China is the Baidu Post Bar, the largest bulletin board community in China but with weak administration. Advertisements can be commonly found on several pertinent post bars at the site post.baidu.com. This system has a keyword-based structure, and there are no other entries to the post bar: if you do not know the keyword to search for, you will not find any malicious entries. The actors within the black market have their own, unique jargon, and thus it is hard for an outsider to find any information about this threat.

The actual trading of virtual assets happens on public market places like Taobao. These very common online business platforms within the WWW are used by the cyber criminals to advertise and sell their goods. After a trade was successful and a Player has bought a virtual good, the money is sent commonly via Alipay. The goods, i.e., the virtual assets, are exchanged through different online mechanisms. They can for example be sent via e-mails or transferred with the help of other services within the WWW.

\subsection{Case Study: Panda Worm}

The most well-known security incident on the Chinese World Wide Web during the year 2007, which also follows the economy model we introduced above, was committed by a Chinese blackhat team. The most important actor is Li Jun, a Virus Writer. He implemented the Panda worm (also known as Worm.Nimaya.w or "panda burning joss stick") based on his experience from implementing several other kinds of malware like for example QQTailEKS, QQpass, Whboy, Whboy 2005. He sold Panda worm to more than 120 blackhats for a price between 500 and 1000 RMB. In December 2006, Li Jun met online with Wang Lei (a Website Master) and Zhang Sun (an Envelopes Stealer). Jun Li and Lei Wang set up several website for hosting the Trojans that are automatically downloaded by users infected with the Panda worm. They sold the website traffic to Zhang Sun, who linked his Web-based Trojans to the websites, thus the victims were infected with several Trojans that are able to steal virtual goods. From the infected machines, the attackers stole the envelopes for online games. Zhang Sun sold the envelopes for a price between 0.9 and 2.5 RMB on the underground market.

The attackers lost control over Panda worm and this resulted in an infection of millions of computers on the Internet in January and February 2007. The losses due to this incident are estimated to be up to 100 million RMB. This huge amount of damage raised the attention of several anti-virus vendors and the police: in February 2007, the criminals were arrested and put into jail. Before they were arrested, all of them made a certain profit: Li Jun made an estimated profit of about 150,000 RMB, Wang Lei 80,000, and Zhang Sun 12,000. In September 2007, Li Jun was sentenced to four years in prison, Wang Lei two and a half years, Zhang Sun two years. Compared to other countries, the imprisonment for crime on the World Wide Web is high in China. For example, the author of Agobot, a powerful bot that also caused high damage, was sentenced by a German court for only 12 months on probation. 


\section{Mechanisms Behind Malicious Websites on the Chinese Web}

\subsection{Overall Technical Flow}

The overall technical flow of the malicious websites phenomenon is shown in Figure 2. The Virus Writers take care of implementing Web-based and conventional Trojans, and use evasion methods to create covert Trojans, and then they sell the malware and evasion service. Website Masters/Crackers betray their customers or crack unsafe websites, and sell the visitor traffic of their own or harvested websites. Envelope Stealers construct a Web-based Trojan network by hosting the bought Web-based and conventional Trojans on compromised computers, and redirect the website visitors to their Web-based Trojans. When the Web-based Trojan network is ready, the victims who visit the malicious websites will be redirected to and exploited by the Web-based Trojans, and infected with further conventional Trojans. These Trojans then steal envelopes and virtual assets from the victim's machine.

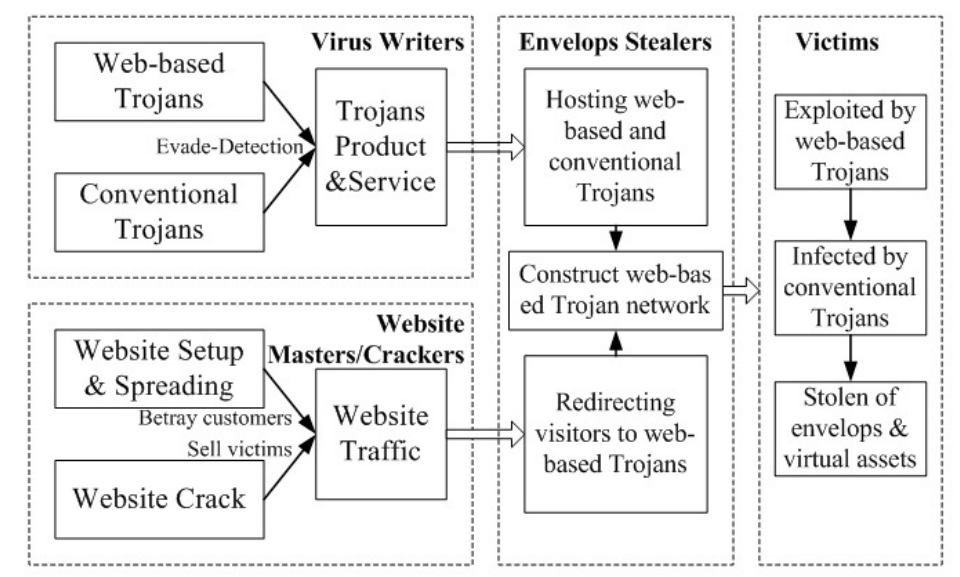

Figure 2: Overview of the overall technical flow for the malicious websites phenomenon

In the following subsections, we discuss in detail the mechanisms used by the Chinese attacker community in the different steps of this technical flow. We focus on the Web-based and conventional Trojans, vulnerabilities used for Web-based Trojans, evasion methods used, strategies for redirecting visitors to Web-based Trojans, and an advanced strategy to build malicious websites using ARP spoofing.

\subsection{Web-based and Conventional Trojans}

There are two different types of Trojans involved in the technical flow of malicious website, i.e., Webbased Trojans and conventional Trojans. Web-based Trojans are used for exploiting the vulnerabilities in the web browser or third-party extensions and injecting the conventional Trojans. The purpose of conventional Trojans is to remotely control the victim's machine and steal envelopes from them.

\subsubsection{Web-based Trojans and Generators}

Web-based Trojans are a kind of client-side attacks, and typically exploit certain system- or applicationlevel vulnerabilities to obtain complete control of the client system once the vulnerable client visits the malicious site. Web-based Trojans are typically implemented in scripting languages including JavaScript and PHP. Virus Writers commonly implement generators which can generate Web-based Trojans automatically and which can be used by other actors in the market without any programming skills.

In the following, we provide a brief example of a Web-based Trojan. A well-known and widely used Web-based Trojan family on the Chinese Web is the MS06-014 Trojan. The source code of the malware is quite easy to understand and it can be downloaded for free from the Internet, e.g., there are about 3,670 
result pages by issuing the search request "MS06-014 Web-based Trojans" (in Chinese) to Baidu Search Engine and about 2,800 pages by issuing "MS06-014 Generators" (in Chinese). Thus it became a primary Web-based Trojan for the attacker community in China during the year 2006, and the exploited MS06-014 vulnerability was entitled the "King Vulnerability for Malicious Websites" accordingly.

To achieve a high exploitation rate, the Virus Writers are always seeking for 0-day or newly disclosed vulnerabilities, and implement new Web-based Trojans exploiting them. They are also targeting Chinesespecific applications and components including Baidu Soba BHO (Browser Help Object), Baofeng media player, PPStream network TV, Xunlei file download software, and others. Since these applications are also very widely installed and used by the Chinese Internet users, and lack an automatic patch update and delivery mechanism, they have become one of the important targets of the Web-based Trojans.

\subsubsection{Conventional Trojans}

Conventional Trojans are also essential tools which contribute to the remote control of the infected "flesh chickens", and the harvesting of stolen envelopes. The history of the conventional Trojans is quite long in the Chinese attacker community. The Virus Writers are constantly developing more powerful Trojans and they struggle against anti-virus vendors and their software.

The most famous and widely used full-functional Trojan after Binghe is the Hack.Huigezi tool, which was developed and maintained by a blackhat group called Huigezi Lab. Although Huigezi was advertised as a network management software, it contains many powerful functions that are beyond the demands of a network administrator, including remote control of large amount of client computers to build botnets, downloading and uploading files, keystroke recording, remote desktop monitoring, setting up proxy servers, and hiding of itself by API hooking and process injecting. Due to its powerful capacities and customized service provided by Huigezi Lab, Huigezi has been widely used by the Chinese blackhat community, also in the area of malicious websites. It was listed as top ten malware for three years from 2004 to 2006 in a row by almost all of the Chinese anti-virus vendors.

There is also a large amount of dedicated stealer Trojans available on the Chinese Web or the underground market. Most of them are QQ stealers and online game stealers driven by the virtual asset market requirements. We can easily find free downloads or advertisements of up to ten different QQ stealer families and stealer Trojans for almost all of the popular online games on the Chinese Web. Other types of Trojans, such as dedicated Web-based bots for click fraud, stealers for online banking and stock trading credentials, are also seen on the Chinese public Web and the underground market.

\subsection{Vulnerabilities Used for Web-based Trojans in China}

Table 1 provides an overview of the number of published system- and application-level vulnerabilities used for Web-based Trojans in China for the years 2003 until 2007. This tables shows two major trends. First, malicious websites are more and more popular, and the number of disclosed and exploited vulnerabilities is increasing. Second, the attackers are moving to the vulnerabilities of common applications instead of system-level vulnerabilities.

\begin{tabular}{|l|r|r|r|}
\hline Year & System Vulnerabilities & Application Vulnerabilities & Total \\
\hline 2003 & 1 & 0 & 1 \\
2004 & 6 & 0 & 6 \\
2005 & 5 & 0 & 5 \\
2006 & 9 & 2 & 11 \\
2007 (January - August) & 8 & 7 & 15 \\
\hline
\end{tabular}

Table 1: Number of system- and application-level vulnerabilities related to the malicious website phenomenon for several years

In Table 2, we provide a more detailed overview of the vulnerabilities for the year 2007. We list the date of the public disclosure, the availability of a patch, and the availability of an exploit for this particular 
vulnerability. The table shows that the time between public disclosure and availability of a patch or exploit is often rather short: for a normal user, the time to patch, i.e., the time between the announcement of a vulnerability and the active exploitation in the wild, is rather short and it is hard to keep up with the latest information. Especially in the area of the WWW, this is an increasing problem since patching third-party applications or browser-addons / ActiveX controls is still not very good automated.

\begin{tabular}{|l|r|r|r|}
\hline Vulnerability ID & Disclosure Date & Patch Available & Exploit Available \\
\hline MS07-004 & 09.01 .2007 & 09.01 .2007 & 16.01 .2007 \\
MS07-009 & 24.10 .2006 & 13.02 .2007 & 26.03 .2007 \\
MS07-017 & 28.03 .2007 & 03.04 .2007 & 08.04 .2007 \\
MS07-020 & 10.04 .2007 & 10.04 .2007 & N/A \\
MS07-027 & 08.05 .2007 & 08.05 .2007 & 10.05 .2007 \\
MS07-033 & 14.03 .2007 & 12.06 .2007 & 14.03 .2007 \\
MS07-035 & 12.06 .2007 & 12.06 .2007 & N/A \\
MS07-045 & 15.08 .2007 & 14.08 .2007 & N/A \\
CVE-2007-3148 & 06.06 .2007 & N/A & 06.06 .2007 \\
CVE-2007-4105 & 02.08 .2007 & 02.08 .2007 & 03.10 .2007 \\
CVE-2007-4748 & 19.08 .2007 & N/A & 31.08 .2007 \\
CVE-2007-4816 & 07.09 .2007 & 20.09 .2007 & N/A \\
CVE-2007-5017 & 19.09 .2007 & N/A & 19.09 .2007 \\
CVE-2007-3296 & 30.05 .2007 & 01.06 .2007 & N/A \\
CVE-2007-5064 & 30.08 .2007 & N/A & 19.09 .2007 \\
\hline
\end{tabular}

Table 2: Time between public disclosure, patch availability, and exploit availability for these vulnerabilities on the Chinese Web for selected vulnerabilities in the year 2007. N/A means that we did not find freely accessible information for patches or exploits.

We also examined the relationship between vulnerabilities and their usage on the Chinese Web. Table 3 list for all vulnerabilities shown in Table 2 the date when an exploit was seen on a malicious website within the Chinese Web and when an exploit for this vulnerability was advertised on the underground black market. For all vulnerabilities we found information about the corresponding exploit available on the Chinese Web and also most of the exploits were advertised on the underground market. This means that the attackers are very active and they also react fast to new vulnerabilities.

\subsection{Evasion Methods used by Trojans}

In this section, we introduce several evasion techniques used by Web-based Trojans to evade detection. We found these methods during our study of the World Wide Web. The methods can be used together to achieve evasion of almost all of the AV engines we tested:

1. Modify the entry point: Change the entry point of the Trojan program using binary analysis and editing tools. This method is simple and practical, but sometimes the sample can still be detected by AV engines.

2. Insert junk instructions to the code: Using binary analysis and editing tools, it is also possible to insert junk instructions which are unrelated to the Trojan functions into the code of Trojans. This is a general method to evade detection by AV engines which is often successful.

3. Use binary packers: Use some rarely seen binary packers to evade the detection by AV engines, or use disguise packers to fool AV engines. These packers can be used nested to achieve better covertness.

4. Obfuscate packer: After packing the Trojan, obfuscate the unpack codes introduced by the packers, or insert junk instructions to the unpack codes. This method can evade the detection of the used packers by AV engines. 


\begin{tabular}{|l|r|r|}
\hline Vulnerability ID & Exploit seen on Chinese Websites & Advertisements on Black Market \\
\hline MS07-004 & 26.01 .2007 & 22.01 .2007 \\
MS07-009 & 28.03 .2007 & N/A \\
MS07-017 & 30.03 .2007 & 10.04 .2007 \\
MS07-020 & 15.09 .2007 & 24.07 .2007 \\
MS07-027 & 30.03 .2007 & 10.04 .2007 \\
MS07-033 & 07.07 .2007 & 13.06 .2007 \\
MS07-035 & 11.07 .2007 & 08.07 .2007 \\
MS07-045 & 02.09 .2007 & 02.09 .2007 \\
CVE-2007-3148 & N/A \\
CVE-2007-4105 & 08.06 .2007 & 23.09 .2007 \\
CVE-2007-4748 & 18.08 .2007 & 08.09 .2007 \\
CVE-2007-4816 & 19.08 .2007 & 08.09 .2007 \\
CVE-2007-5017 & 06.09 .2007 & N/A \\
CVE-2007-3296 & 26.09 .2007 & 28.06 .2007 \\
CVE-2007-5064 & 25.06 .2007 & 14.09 .2007 \\
\hline
\end{tabular}

Table 3: Point in time for Trojans available on WWW in China and black market advertisements for selected vulnerabilities from Table 2. N/A means that we did not find any advertisements on the underground black market.

5. Identify the signatures, modify the codes to eliminate them: Identify the signatures used by AV engines to detect the Trojan and then modify the related codes to eliminate signatures. Detailed techniques include change the upper case and lower case of some string based signatures, equivalent instructions replacement, change the sequence of unrelated instructions, and obfuscate the sequence of codes by inserting different kinds of jump instructions.

We also found several specific evasion methods used by Web-based Trojans:

1. Obfuscate the source codes of the Web-based Trojan: The commonly used tool is screnc.exe, a source code obfuscation tool provided by Microsoft. The obfuscated Web-based Trojan can evade the detection of most $\mathrm{AV}$ engines while keeping the functions intact. Customized encryption tools based on JavaScript (although simple) are used and have achieved better effects.

2. Obfuscate the source codes of the Web-based Trojan by encoding: Change the uppercase and lowercase of the source codes, and replace some sensitive strings with hex- or unicode-encodings. While being simple, this can also be used to evade $\mathrm{AV}$ detection.

3. Divide the source codes into pieces: Divide the source codes of the Web-based Trojan into multiple files, and use \#include to combine them to fully functional Trojans.

4. Fool the AV engines by simply changing the file mask: Change the file mask of the Web-based Trojan from . asp / . aspx/.php/.jsp/.js to others, such as . jpeg or . png.

5. Obfuscate the structure of the Trojan by including some signatures of other file types: Include some signatures of other file types (. jpeg, mdb access database file) in the beginning of the Trojan, while keeping the functions correct.

During our measurement study, which we introduce in Section 5, we found that almost all of the inthe-wild Web-based Trojans are adopting some kinds of these evasion methods to achieve evasion of the major $\mathrm{AV}$ engines. We present measurement results of this phenomenon in a later section. 


\subsection{Strategies for Redirecting Visitors to Web-based Trojans}

To redirect the visitors of the trojanized websites to the actual Web-based Trojan, attackers are typically using one of the following three categories of strategies.

\subsubsection{Embedded HTML Tags}

The first category uses embedded HTML tags such as iframe, frame, and others, to embed the Webbased Trojan into the source code of the website. In order to achieve better covertness and flexibility, the attackers often introduce some intermediary stepping stones and dispatchers to build complex and obfuscated Trojan networks, by recursively using embedded tags and obfuscating the destination location.

The most used tag in the wild for redirection is iframe: the purpose of this HTML element is to create an inline frame that contains and displays another document. When the including page is opened, the included document is displayed in the inline frame. The attackers take advantage of this characteristic to include the Web-based Trojan directly or recursively, but always set the iframe to be invisible. This can be easily achieved by setting the height or width of the iframe to zero or a very small value, for example:

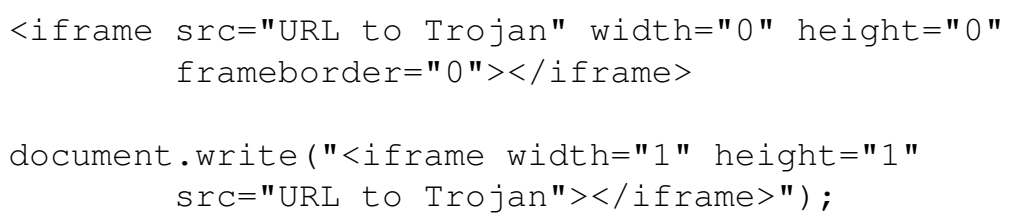

The frame tag can be also used to including Web-based Trojans, but it is a little bothering to define a frameset and include the URL to Web-based Trojan in an invisible frame, so it is rarely used by attackers in the wild. Other strategies belonging to this category include using the body on load event to load Web-based Trojan, and injecting links to Web-based Trojan into CSS and various other tags.

\subsubsection{Malicious Scripts}

The second and also popular category uses the script tag to include Web-based Trojan scripting or redirector scripting, which are often XSS (Cross-Site Scripting) vulnerabilities. The redirector scripting typically uses document.write to generate an iframe tag which includes the Web-based Trojan or further stepping stones, or rarely seen windows. open function to obviously popup a new HTML window to perform exploitation.

\subsubsection{Embedded Objects}

The third category of strategies for including Web-based Trojan is based on the embedded object tag for activating third-party applications (e.g., Flash or Baofeng media player) or Browser Helper Objects (BHOs) to display the embedded object. When vulnerabilities in these applications and BHOs are found, attackers then use this strategy to inject the carefully constructed objects to the vulnerable applications, which exploit them in order to remotely execute code on the victim's machine.

A classical example belonging to this category is a technique widely used by Chinese attackers during the last year to include Web-based Trojans. This techniques is based on an exploit of a vulnerability within Internet Explorer (MS06-021): the attackers can generate a malicious Flash file by injecting the URL to Web-based Trojans into a normal, benign SWF Flash file, and then they can include this specially prepared Flash file in a website which they control or other well-known websites which provide the Flash uploading and browsing service. When the malicious Flash file is displayed by a vulnerable version of Internet Explorer, the visiting computers are then attacked and redirected to execute the Web-based Trojans. The machine of the visitor is then compromised. 


\subsection{Advanced Malicious Website Strategy using ARP Spoofing}

Besides the formerly introduced strategies, there is another advanced strategy to build malicious website when the attackers can not gain control of the target website. This method does not actually compromise the target website, which is presumably well protected, especially the most well-known websites with large amounts of visitors. The attacker uses ARP spoofing in order to act as a Man-in-the-Middle, and hijacks all of the traffic from and to the victims in the same Ethernet subnet. The attacker then injects malicious code into the HTML responses from specific domain (i.e., the target website), or all of the web traffic, to achieve virtual malicious websites. When the attacker has compromised server within the same collision domain as the target site, he can then inject malicious redirection code into all of the HTML responses from the target website, and harm all of the visitors who use vulnerable systems. The ARP spoofing attack was observed in the wild: for example, the Norton China website, which belongs to a larger community, was attacked using ARP spoofing at October 9, 2007 [4]. It really raises severe threats to the WWW, especially in China, since a majority of Chinese websites are hosted by ISP or web hosting providers densely in their subnets.

The most widely used ARP spoofing tool for malicious website in China is a little command-line hack tool called zxarps, which is written by a coder named LZX in the Ph4ntOm Security Team. An example command to compromised a website using this tool is provided in the following example:

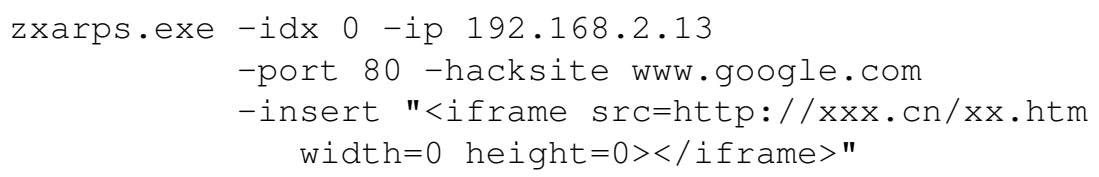

\section{Measurements and Results}

To understand the situation posed by the malicious websites phenomenon and the prevalence of the underground economy on the Chinese Web, we performed a comprehensive measurement study in October 2007. Our measurement setup covers the observation and analysis on the underground black market, the analysis on the public marketplace for the virtual assets trading, and a detailed and in-depth evaluation of the threats raised by malicious websites to the normal Chinese Internet users.

\subsection{Measurements on the Underground Black Market}

Unlike the US or EU blackhats communities, Chinese blackhats are typically not familiar with IRC (Internet Relay Chat). They typically use bulletin board systems on the Web or IM software like QQ to communicate with each other. Orthogonal to a study on the underground black market located within IRC networks [5], we measure the Chinese-specific underground black market on the Web. We focus on the most important part located at post.baidu.com, the largest bulletin board community in China. We crawled the portal and stored all posts and replies posted on some certain post bars which are all dedicated for the underground black market on this particular website. The post bars we examined include Traffic bar, Trojans bar, Web-based Trojans bar, Wangma bar (acronyms of Web-based Trojans in Chinese), Box bar, Huigezi bar, Trojanized websites bar, and Envelopes bar.

Each post and reply in the bar contains a title, information about the poster, post time, and the actual content. If the poster is not registered, the poster field is filled with the Class C IP range from where the poster connects to the server. Although it is possible that one person connects to the server from different IP ranges, we can still use this information to represent the poster since this situation happens only rarely due to the lack of IP addresses in China. For each of the IP addresses, we queried the geographical location of the poster using ChunZhen, a well known IP2Location library within the Chinese Internet community. Our measurements show that 23,606 distinct posters were involved in the underground market between January 2006 and September 2007. In total, they posted 90,679 posts or replies during this period of time. As shown in Figure 3 and 4, the numbers of posts published on these post bars per month are increasing over time, as well as the numbers of posters. At the peak point in August 2007, almost 3,500 
posters published nearly 14,000 posts on the underground market, which shows that the underground black market is quite active in China. We also provide an overview of the province distribution of the underground market participants in Table 4, based on the location query results of the posters.

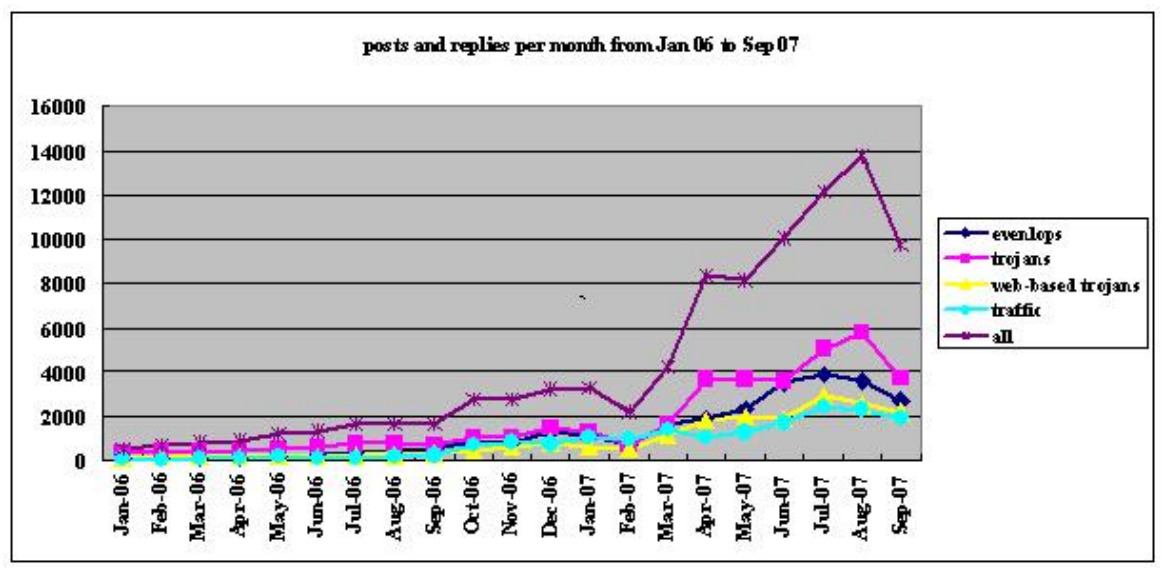

Figure 3: Posts and replies per month from January 2006 to September 2007

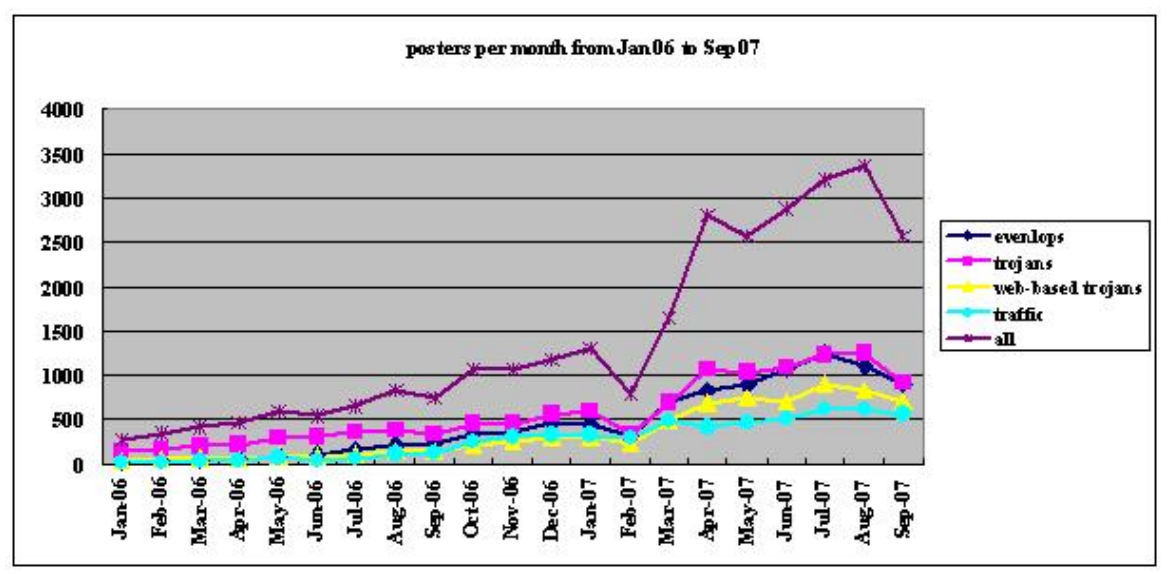

Figure 4: Posters per month from January 2006 to September 2007

\subsection{Measurements on the Public Virtual Assets Marketplace}

We also studied the public assets marketplace visible via the Taobao online business platform. We found 42,561 online shops in total, and 34,450 of them had bargained deals successfully. Furthermore, our measurements show that there are a total of 1,220,181 virtual goods in all of these shops available, which means that each shop has on average 28 goods.

To estimate the market value of the whole virtual asset exchange market on the Taobao platform, we crawled the credit history pages of all the shops. From these pages it is possible to retrieve more information about the overall trading activity during different periods, including in the last week, the last month, the last six months, and the whole lifetime of the online shop. Furthermore, this page also contains the geographical location of the shop owner. Based on this information, we calculated that there were a total of 8,907,568 successful deals within the last six months. We also examined all of the successful deals in one typical shop to estimate the value per deal, which resulted in an average total price of 12.56 RMB. Based on these values, we can estimate that the market value of the virtual asset exchange is about 


\begin{tabular}{|l|r|r|}
\hline Province & \# Posters & Percentage \\
\hline Guangdong & 1,939 & $10.75 \%$ \\
Shangdong & 1,460 & $8.10 \%$ \\
Zhejiang & 1,271 & $7.05 \%$ \\
Jiangsu & 1,126 & $6.24 \%$ \\
Hebei & 965 & $5.35 \%$ \\
Liaoning & 964 & $5.35 \%$ \\
Beijing & 910 & $5.05 \%$ \\
Hubei & 837 & $4.64 \%$ \\
Henan & 837 & $4.64 \%$ \\
Fujian & 820 & $4.55 \%$ \\
Others & 6,904 & $38.29 \%$ \\
\hline
\end{tabular}

Table 4: Province distribution of black market participants

223 million RMB in total, on the Taobao platform. Taking into account that the virtual asset exchange can also take place on PaiPai, eBay, and thousands of smaller, but dedicated online markets and shops, we conclude that there is a prospering virtual asset industry. These numbers also explain why the underground market is so attractive for malicious attackers, especially since the legal situation in China does not take cyber-crime related to stealing of virtual assets into account.

Based on the data collected on Taobao, we can also generate a province distribution of the successful virtual asset deals during the last six months as shown in Table 5. We can compare this with the distribution of black market posters shown in the previous section. Six provinces, including Guangdong, Zhejiang, Jiangsu, Hubei, Beijing, and Shangdong, are in both of the top ten tables, which reflects the strong relations between the public virtual asset market place and the underground black market.

\begin{tabular}{|l|r|r|}
\hline Province & \# Deals & Percentage \\
\hline Guangdong & 905219 & $10.16 \%$ \\
Zhejiang & 898042 & $10.08 \%$ \\
Jiangsu & 806167 & $9.05 \%$ \\
Shanghai & 740390 & $8.31 \%$ \\
Hubei & 581767 & $6.53 \%$ \\
Beijing & 544457 & $6.11 \%$ \\
Fujian & 429652 & $4.82 \%$ \\
Shangdong & 398365 & $4.47 \%$ \\
Jiangxi & 350297 & $3.93 \%$ \\
Sichuan & 329375 & $3.70 \%$ \\
Others & 2923837 & $32.82 \%$ \\
\hline
\end{tabular}

Table 5: Province distribution of Taobao virtual asset marketplace deals

\subsection{Malicious Websites on the Chinese Web}

In this work, we are not only interested in finding malicious websites and analyzing them in-depth, but also in gaining an overall understanding about how much this phenomenon threatens the normal Chinese Internet users. We thus want to examine the WWW in China and try to gain a better understanding of the malicious "corners" within the Web. In the following paragraphs, we describe our measurement setup and present the results of our measurement study. 


\subsubsection{Measurement Setup}

According to the status report published by China Internet Network Information Center (CNNIC), there are a total of 1.31 million websites on the Chinese WWW in June 2007 [2]. Since checking the whole content of the Chinese Web is infeasible due to the size of the Web, we need to find a way to efficiently inspect a representative part of it. We thus need a good sampling strategy in order to find the parts of the Web that are most commonly accessed by normal Internet users. According to the same report by CNNIC, about 75\% percent of the Internet users within China use search engines to find their information and target websites on the WWW. To sample the Chinese WWW effectively and focus on the major threats to the majority of Chinese Internet users, we thus use search engines to find the starting points of our experiments. We used two input sources to obtain the most commonly used keywords by Chinese Internet users to find their information: first, we used the Baidu top search keywords list provided at http://top.baidu.com. Second, we included the Google Chinese ReBang ("Top hot search keywords") provided at http://www. google.cn/rebang/home. Using the combined list of both inputs, we then categorized the searched websites into the following twelve categories: portal/navigation, movie/TV, game, news/information, sport/entertainment, free download, e-business, industry information, chat/virtual society, e-finance, warez, and user content. By issuing the most commonly used keywords for the specific content area to the Baidu and Google search engines, we obtained about 145,000 domain names. This set of sites represents our sampling set. Furthermore, we built a blacklist category containing the recently reported malicious websites by the Chinese Internet community during a one-week period before our measurements. We inspected all these websites to examine whether they are malicious or not. We also categorized the websites to learn whether there are some specific areas on Chinese WWW that are more risky than others.

To actually inspect these websites, we developed a client honeypot that is capable of efficiently examining whether or not a given website is malicious. In contrast to previous work in this area, we split the task in two steps: in the first step, we examine websites with a high-interaction honeypot. A honeypot is a system which is intended to be probed, accessed, or compromised [7]. High-interaction means in this context that we use a real system for performing the analysis: The basic idea is to execute a webbrowser within a honeypot environment, automatically "surf" websites, and closely observe all activities on the honeypot. If we open a web page within the honeypot and this website exploits a vulnerability in our browser, we can detect this malicious behavior and issue an alert. In a second step, we use a lowinteraction honeypot: instead of using a real system, we use a web crawler to automatically download and analyze larger amounts of data. As starting point for these crawls we use malicious websites identified in the first step. This two-tier architecture helps us to scale the system and analyze content in more depth.

Our high-interaction client honeypot is built upon our formerly developed automatic malware monitoring and analysis tool called MwSniffer, and our automatic malware collecting tool called HoneyBow [13]. MwSniffer executes the to be analyzed malware sample within an instrumented Windows environment and observes during runtime the behavior of the sample. The tool is capable of monitoring the activity of the sample on the system and monitors various aspects, including:

- Creation of processes to detect newly running executables

- File system modifications to detect infected and extracted malicious files, including rootkits

- Windows registry modifications to detect registry keys added or modified by malware

- Dumping the network traffic related to the malware execution and parsing the captured traffic in order to extract full protocol information for common application protocols including HTTP, FTP, IRC, SMTP, and POP3

- New listening network ports to find out backdoors opened by the analyzed binary

The second tool, HoneyBow, is intended to fetch samples of autonomous spreading malware in an automated manner [13]: we monitor the network traffic between the honeypots and the Internet and extract all executables that pass by, since these binaries are presumably malicious: if a website exploits 
a vulnerability in our honeypot browser and installs a malware binary on this system, we can monitor the infection step when watching all network packets since we can then detect the actual transfer of the binary. In our setup, we integrate HoneyBow into the client honeypot in order to capture these samples.

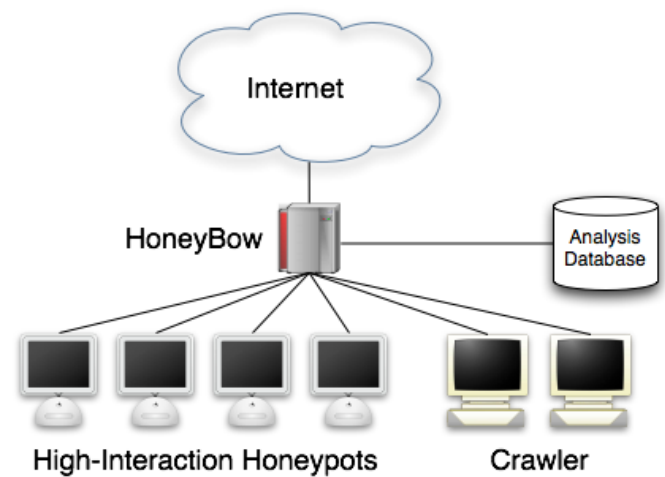

Figure 5: Schematic overview of the client honeypot setup

In Figure 5, we provide a schematic overview of our client honeypot. We run the honeypots within VMware virtual machines. Each instance of the client honeypot is based on a default Windows XP SP2 Chinese version. Mwsniffer is executed on each of these machines in order to detect changes of the system state. The honeypots are scheduled to fetch a website domain name from a central database, and browse the website using Internet Explorer 6. MwSniffer monitors the changes of the system and stores the results back into the central database. After a time-out of two minutes, which is enough time for opening the index page of the website and monitoring of an exploit, the virtual machine is reverted to a clean state and continues its next check of the remaining websites.

Based on the monitoring and analysis reports generated by MwSniffer during the check period, we can then find out which websites are malicious and cause changes of the system state. We need to take care of false positives: we exclude in the analysis process authorized, i.e., benign, state changes such as write activity within the browser cache or registry changes due to Internet Explorer startup. All other changes are suspicious and indicate a website that we need to analyze in more detail: presumably we have found a malicious website that exploited a vulnerability in our client honeypot browser and caused system state changes of the analysis system.

To analyze the malicious websites in-depth, we use in a second stage low-interaction honeypots to crawl malicious sites. This combination of high- and low-interaction honeypot allows us to build a scalable system and differentiates our implementation from previous work in this area. We developed a lightweight crawler which can crawl the index page of confirmed malicious websites and its recursively linked pages via embed tags including iframe, script, frame, and other tags (Crawler in Figure 5). We choose these tags based on a manual analysis of malicious web pages and choose tags which are popularly used by the malicious attackers to redirect the innocent website visitors to their Web-based Trojan hosts. The embed link relations between the pages were also recorded and inserted into the database for further analysis. This data is the basis of our link analysis of malicious websites.

The end-point Web-based Trojan pages were also crawled and stored. Because of the code obfuscation commonly adopted by the Web-based Trojans to evade common detection schemes, our crawler currently lacks the capability to normalize the codes and download the further injected malware. But with the help of our HoneyBow and MwSniffer tools, we can fetch all of the necessary raw data for further analysis and case treatment, including malicious website behavior reports generated by MwSniffer, raw network packet dumps recorded by MwSniffer, the content of pages and their link relations crawled by the crawler, and the injecting Trojans fetched by HoneyBow. As part of our future work, we plan to incorporate these data to further automate the analysis of malicious websites. 


\subsubsection{Malicious Websites}

Based on the measurement setup we introduced in the former subsection, we identified a total of 2,149 malicious websites from 144,587 distinct hosts which represent the most commonly visited websites by normal Chinese Internet users. Table 6 provides an overview of the measurement results for the twelve different categories, the blacklist, and the total sites. We found that the categories including freedownload, sport/entertainment, movie/TV and chat/virtual society are more risky than others, which is consistent with our anticipation. The results also reveal that all categories contain a significant amount of malicious content: this is an important discovery as it means any Chinese Internet user accessing the web is at risk, regardless of the type of content they browse. Given the fact that all these sites were found using a search engine, this proofs that the threat is significant.

\begin{tabular}{|l|r|r|r|r|}
\hline Category & Keywords & Inspected & Malicious & $\%$ \\
\hline Free Download & 22 & 20,547 & 394 & 1.92 \\
Sport/Entertainment & 31 & 27,649 & 520 & 1.88 \\
Movie/TV & 25 & 23,472 & 423 & 1.84 \\
Chat/Virtual Society & 6 & 8,115 & 140 & 1.73 \\
Game & 23 & 20,105 & 269 & 1.34 \\
News/Information & 29 & 36,700 & 459 & 1.25 \\
Warez & 14 & 13,237 & 164 & 1.24 \\
Portal/Navigation & 6 & 8,829 & 106 & 1.20 \\
Industry Info & 17 & 20,518 & 246 & 1.20 \\
e-Finance & 15 & 19,138 & 139 & 0.73 \\
e-Business & 6 & 9,799 & 64 & 0.65 \\
User Content & 6 & 7,402 & 33 & 0.45 \\
\hline Total with overlaps & 200 & 215,511 & 2,965 & 1.38 \\
Distinct Total & 200 & 144,587 & 2,149 & 1.49 \\
\hline \hline Blacklist & N/A & 796 & 28 & 3.52 \\
\hline
\end{tabular}

Table 6: Measurement results for malicious websites on the Chinese Web

The measurement results for the different categories reveal that different parts of the Web have a different degree of maliciousness: we found that user content is only malicious in $0.45 \%$ of the sites, while free download sites have a significant higher chance of hosting malicious content.

\subsubsection{Link analysis}

Based on the collected data, we can also generate interrelations between different malicious websites. This allows us to connect different attacks and we can learn more about relations between the involved domains. We have generated several graphs revealing the link relations between the involved domains, redirectors, and the hosts of the Web-based Trojans discovered during our measurement. In Figure 6, we show an example of such a graph, in which we see that different domains are connected via malicious content included as an embedded link.

Similar to the node ranking strategy adopted by HoneyMonkey [12], we also assign the incoming and outgoing ranks to all of the hosts in the overall graph. We focus on the top-level domain names and not the low-level redirection links. Our strategy can reflect the popularity of the exploiters more precisely than the graphs generated by HoneyMonkey. Ordered by the incoming ranks, we were able to list the top malicious sites, together with the number of directly linked malicious websites or redirectors. Shutting down these domains would effectively lower the total amount of malicious websites on the Chinese Web since these domains are one of the root causes.

We also analyzed the top exploiter in more depth, and found out that 490 malicious websites $(22.8 \%$ of total) located at 206 different top domains redirected their traffic to this particular attacker. The links 


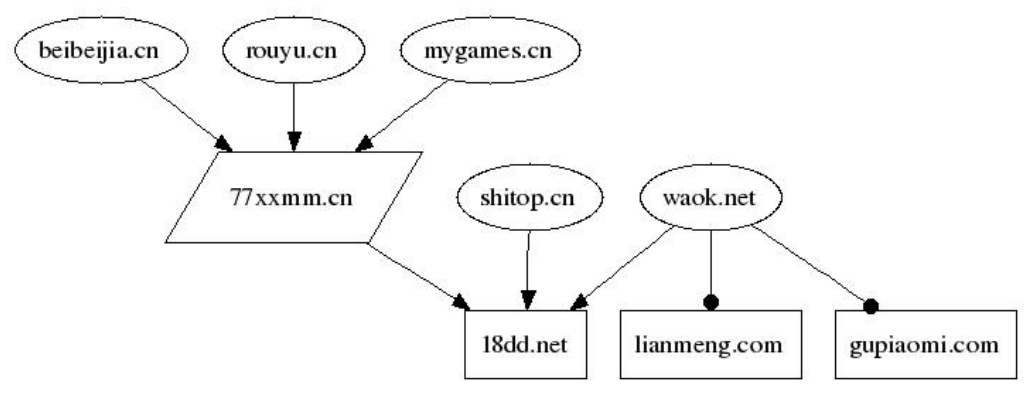

Figure 6: Example of link analysis of malicious websites. Trojanized websites are drawn as ellipse, the redirector websites are drawn as parallelogram, and the exploit-hosting websites are drawn as a box.

were either directly or via 26 redirectors. The attacker used heavily obfuscated hosted dispatcher scripts and Web-based Trojans to evade detection. In total, we found 21 different executables being used in this attack, the majority of them are identified as online game stealers.

An interesting observation we can find from the link analysis is that the attackers register large amounts of . cn domains and use them for their attacks. This is specific to the Chinese Web since starting in March 2007, the register fee for . $\mathrm{cn}$ domains is only 1 RMB for the first year to encourage the development of Chinese Web. Attacker abuse this in order to have many domains which redirect innocent Internet users to malicious websites. To even enhance the effectiveness of this strategy, the attackers generate many second-level domain names on these cheap domains. The domain names themselves seem to be generated in a random fashion and follow no obvious pattern.

\subsubsection{AV Detection Rates}

In order to study how good an average Internet user is protected against this threat with the help of anti-virus (AV) engines, we also scanned every collected samples with MWScanner. This is a tool we developed that combines nine common AV engines, to identify known malware variations and families, and to examine the detection rates of these AV engines. Table 7 provides an overview of the detection rates for different $\mathrm{AV}$ engines. If the $\mathrm{AV}$ engine detects a malicious file from the downloaded case data (including web-based and conventional Trojans), then we count this as a case detection. If the AV engine detects a malicious executable (PE file format) or a malicious sites, we count it as a conventional Trojan detection. Finally, if the AV engine detects a malicious non-executable (not PE file format) from the case data, then we count it as a web-based Trojan detection. For the sake of brevity, we just show the detection rates for the best international and local AV engine.

\begin{tabular}{|l|r|r|r|}
\hline AV Engine & Case & Web-based & Conventional \\
\hline Best International & $86.1 \%$ & $25.4 \%$ & $83.6 \%$ \\
Best Local & $88.7 \%$ & $36.7 \%$ & $84.7 \%$ \\
\hline
\end{tabular}

Table 7: Detection rates for malicious websites as a whole case, and the Web-based / conventional Trojan

Our measurements show that all of the AV engines achieve poor detection rates for the Web-based Trojans, much worse than the detection rates for conventional Trojans. This is presumably mainly due to the heavy obfuscation methods used by the attackers to evade detection, and it seems that the AV vendors have not paid enough attention to the threats posed by malicious websites. 


\section{Conclusion}

In this paper, we studied several aspects of malicious activities within the World Wide Web. First, we introduced a model of the underground black market which describes the interaction of the different actors within the market. This model is based on empirical data collected within China. Based on this model, we presented the first empirical measurements of malicious websites within the Chinese part of the World Wide Web. We studied the activities of attackers, how they trade the virtual goods (e.g., exploits or envelopes), and where they are located. Furthermore, we also examined malicious content within the Chinese Web. We combine high- and low-interaction honeypots to study whether or not a given sites contains malicious content.

\section{Acknowledgments}

The research was supported in part by the 863 High-Tech Research and Development Program of China under Grant No. 2006AA01Z445, and Chinese Information Security Research Plan under Grant No. 2006A30. The views and conclusions contained here are those of the authors and should not be interpreted as representing the official policies or endorsements of the C.N. Government or any of its agencies.

\section{References}

[1] Alexa, the Web Information Company. Global Top 500 Sites, September 2007. http: / / a lexa . com/site/ds/top_sites?ts_mode=global.

[2] China Internet Network Information Center (CNNIC). 20th Statistical Reports on the Internet Development in China, July 2007. http://cnnic.cn/download/2007/ 20 thCNNICreport-en.pdf.

[3] T. Cymru. Cybercrime: an epidemic. ACM Quеие, 4(9):24-35, 2006.

[4] DON. ARP spoofing attack against Norton China, October 2007. http://www. nod32club. com/forum/thread-27215-1-1.html.

[5] J. Franklin, V. Paxson, A. Perrig, and S. Savage. An inquiry into the nature and causes of the wealth of internet miscreants. In Proceedings of CCS'07, 2007.

[6] A. Moshchuk, T. Bragin, S. D. Gribble, and H. M. Levy. A crawler-based study of spyware in the web. In Proceedings of 13th Network and Distributed System Security Symposium (NDSS'06), 2006.

[7] N. Provos and T. Holz. Virtual Honeypots: From Botnet Tracking to Intrusion Detection. AddisonWesley, July 2007.

[8] N. Provos, D. McNamee, P. Mavrommatis, K. Wang, and N. Modadugu. The ghost in the browser: Analysis of web-based malware. In Proceedings of HotBots 2007, 2007.

[9] The Honeynet Project. Know Your Enemy: Malicious Web Servers, August 2007. http: / / www . honeynet.org/papers/mws/.

[10] The Honeynet Project. Know Your Enemy: Web Application Threats, February 2007. http: //www. honeynet.org/papers/webapp/.

[11] R. Thomas and J. Martin. The underground economy: Priceless. USENIX ;login:, 31(6):7-16, 2006.

[12] Y.-M. Wang, D. Beck, X. Jiang, R. Roussev, C. Verbowski, S. Chen, and S. T. King. Automated web patrol with strider honeymonkeys: Finding web sites that exploit browser vulnerabilities. In Proceedings of 13th Network and Distributed System Security Symposium (NDSS'06), 2006.

[13] J. Zhuge, T. Holz, X. Han, C. Song, and W. Zou. Collecting autonomous spreading malware using high-interaction honeypots. In Proceedings of ICICS'07, 2007. 